\title{
Das Projekt Fünf lyrische Pantomimen
}

\author{
Ein Bericht über den Versuch, lyrische Texte und szenisches \\ Gestalten in den DaF-Unterricht für brasilianische Studie- \\ rende zu integrieren
}

\section{Christoph Schamm}

\section{Zusammenfassung}

Gedichte fristen im DaF-Unterricht ein Schattendasein. Der vorliegende Aufsatz berichtet von einem Projekt mit brasilianischen Studierenden des Faches Deutsch, das lyrische Texte gewinnbringend in die Sprachvermittlung integrieren sollte: Durch Rezitation und szenisches Spiel wurden den Lernenden neben den Gedichten Aspekte der deutschsprachigen Kultur näher gebracht. Neben dem Bericht über die konkrete Projektarbeit reflektiert der Aufsatz über die Bedeutung von Lyrik im interkulturell perspektivierten DaF-Unterricht.

\section{Vorbemerkung}

Der wesentliche Inhalt dieses Aufsatzes besteht in meinem Bericht über ein Projekt mit dem Titel Fünf lyrische Pantomimen, das ich mit einer Gruppe von Studierenden des Faches Deutsch im ersten Semester des akademischen Jahres 2008 an der Universidade Federal do Rio Grande do Sul (UFRGS) in Porto Alegre, Brasilien, entwickelt habe. Dabei handelte es sich um die Rezitation mehrerer deutschsprachiger Gedichte, die simultan durch stummes Theaterspiel begleitet wurden. Dieses Projekt verfolgte mehrere didaktische Ziele: Zum einen sollte den Studierenden ein Zugang zu lyrischen Texten ermöglicht werden, wie er so unmittelbar in gemeinsamen Lektüren in regulären Deutschstunden nicht geschaffen werden kann. Überdies sollte erprobt werden, ob die detaillierte Auseinandersetzung mit den lyrischen Tex- ten das Interesse der Studierenden für komplexe Sprachstrukturen und gesuchte Lexik wecken kann, ferner verstand sich das Projekt als konkrete Auseinandersetzung mit Aspekten deutscher Kultur. Es sah sich damit einer Konzeption des DaF-Unterrichts verpflichtet, die unter der Lehre der deutschen Sprache weit mehr als nur das Erarbeiten der vier Sprachfertigkeiten versteht.

Neben den theoretischen Grundgedanken, auf die ich im ersten Teil des Aufsatzes näher eingehen will, war die konkrete Entwicklung des Projektes Fünf lyrische Pantomimen von meinen Arbeitsbedingungen an der Deutsch-Abteilung der UFRGS bestimmt. Meine brasilianischen Kollegen und ich beschlossen zu Beginn des Semesters 1/2008, die Aktivität unserer Abteilung einem breiteren Publikum von Dozenten und Studierenden der geisteswissenschaftlichen Fakultät zu präsen- 
tieren. Daher veranstalteten wir am 16. Mai 2008 einen Tag der Germanistik - auf portugiesisch: Jornada de Estudos Germanísticos - mit Vorträgen und Diskussionen über die Arbeit unserer verschiedenen Forschungsgruppen. Zugegeben: Der Wunsch meiner Kollegen, ich möge gemeinsam mit Studierenden eine »apresentação artística «, d.h. eine künstlerische Darbietung «, zu unserer Veranstaltung beisteuern, kam überraschend: Ich bin kein Theaterpädagoge. Ich akzeptierte jedoch die Herausforderung, innerhalb von acht Wochen ein Konzept für eine etwa 45minütige szenische Präsentation zu erarbeiten und es mit neun interessierten Studierenden zu verwirklichen. Das Projekt soll auf den folgenden Seiten in Theorie und Praxis beschrieben werden.

\section{Vorarbeit zum Projekt}

\subsection{Lyrische Texte im DaF-Unterricht}

Das Komische hat in der deutschsprachigen Lyrik seinen festen Platz. Diese Aussage widerspricht dem verbreiteten Vorurteil, dass deutsche Lyrik meist ernst und gedankenschwer, im Grunde also Philosophie in Versen sei. Natürlich ist jedes Vorurteil per definitionem falsch; dies liegt in der Natur des Vorurteils. Wir Deutschen sind an dieser Lesart unserer Literatur nicht ganz unschuldig. Steffen Jacobs legt im Nachwort seines Sammelbandes komischer Gedichte überzeugend dar, dass dieser lyrischen Untergattung in traditionsreichen Anthologien wie Reiners Ewigem Brunnen allenfalls Nischen zugewiesen worden sind (vgl. Jacobs 2004: 819).

Das zitierte Vorurteil ist jedoch sogar in zweifacher Hinsicht völliger Unsinn.
Denn zum einen sind Humor und Satire, Absurdität und Nonsens in der deutschsprachigen Lyrik seit langem etabliert, zum anderen schließen Philosophie und Komik einander nicht notwendig aus. Schon gar nicht im Gedicht. Dies führen uns Texte von Wilhelm Busch, Paul Scheerbart, Christian Morgenstern, Joachim Ringelnatz, Klabund, Erich Kästner, Kurt Tucholsky oder, in jüngerer Zeit, Ernst Jandl, Peter Rühmkorf und Robert Gernhardt deutlich vor Augen. Sie alle sind Repräsentanten einer lyrischen Untergattung, die eine lange, ausgeprägte Traditionslinie und großen Facettenreichtum aufweist. Das Komische kann im Dienst der politischen Satire und Sozialkritik stehen, indem es mehr oder weniger aktuelle Probleme durch Überzeichnung ins Lächerliche zieht; es kann jedoch auch zeitlose menschliche Schwächen von der heiteren Seite nehmen. Nicht selten werden andere lyrische Texte zur Zielscheibe des Spottes: Parodien, Travestien und Persiflagen entlarven die hohle Rhetorik von überkommenen Gedichtformen und Stilrichtungen. Darüber hinaus umfasst das Spektrum Sprachexperimente, die mit dem Rohmaterial der Lyrik, den Worten und Lauten, komische Wirkungen erzeugen. Man muss nicht extra erwähnen, dass gerade die letztere Kategorie beeindruckende Fusionen von Schwach- und Tiefsinn erzeugt.

Der didaktische Nutzen von Liedern für den DaF-Unterricht ist schon lange unumstritten. Auf den CDs in den Buchdeckeln der Lehrwerke werden nicht nur Grußformeln gerappt, es sind auch Popsongs, z. B. von Grönemeyer, enthalten, ${ }^{1}$ damit sich die Lernenden mit Hilfe ihrer

1 Diese Beispiele für Popmusik als didaktisches Mittel im DaF-Unterricht stammen aus dem Lehrwerk Tangram. Deutsch als Fremdsprache; ich beziehe mich hier auf den »Tag wie geht's«-Rap (vgl. Tangram 2002: 1A, 11) und Herbert Grönemeyers Lied Currywurst (vgl. Tangram 2002: 1B, 126). 
Melodien und Rhythmen Sprachstrukturen leichter einprägen. Das Gedicht hingegen fristet ein Schattendasein. Es gilt als schwer zugänglicher Text, der aufgrund des Versmaßes komplizierte grammatische Formen benötigt, ganz zu schweigen von gesuchtem Vokabular. Esselborn spricht sich im Handbuch interkulturelle Germanistik zwar für den Einsatz von Gedichten im DaF-Unterricht aus, beschränkt diesen aber im selben Atemzug auf die »Alltagslyrik « der 70er Jahre, nicht näher definierte Lieder und Songs sowie »die einfachen Formen der konkreten Poesie zur Auflockerung und zu einem freieren Umgang mit der fremden Sprache " (Esselborn 2003: 485). Die prinzipielle Aufgeschlossenheit scheint also durch bestimmte Vorbehalte relativiert. Diese Einwände gegen die Brauchbarkeit der Lyrik im DaF-Unterricht sind in vielen Fällen berechtigt und lassen sich nicht einfach von der Hand weisen. Ältere Gedichte entsprechen weder unserer heutigen, noch, in vielen Fällen, der zeitgenössischen Standardsprache; neuere verweigern sich häufig durch beabsichtigte Sinndunkelheit dem Verständnis des Lesers; alle miteinander neigen sie wegen des Zwanges zu Versmaß und Endreim zu komplizierter Lexik und Syntax. Für Lese- und Hörverstehensübungen, zumal in der Grundstufe, scheinen sie daher kaum geeignet. Dennoch soll in diesem Aufsatz eine Lanze für den didaktischen Einsatz des Gedichts im DaFUnterricht gebrochen werden.

Der cultural turn in den Humanwissenschaften hat insbesondere die Literaturbetrachtung in neue Richtungen gelenkt, indem die Textinterpreten ihr Interesse auf die Erschließung von eigener oder fremder Kultur ausgerichtet haben. Man mag dagegen einwenden, dass die Literaturwissenschaft dieses Ziel von jeher verfolgt habe, sofern sie sich davon wertvolle Erkenntnisse versprochen habe.
Neuerdings laufe sie jedoch Gefahr, ihr angestammtes Terrain aus den Augen zu verlieren. Der euphorische Aufbruch ins Eldorado der Kulturwissenschaften führe letztlich nur zum Verlust des gesicherten Grundwissens und der bewährten Methoden. Die wesentlichen Einwände gegen die kulturalistische Wende in den Philologien lassen sich, knapp und kompetent zusammengefasst, in Nünnings Plädoyer für eine mentalitätsgeschichtlich orientierte Kulturwissenschaft nachlesen (vgl. Nünning 2004: 173-177). Es sei daher besser, wenn die Literaturwissenschaft ihrer traditionellen Selbstdefinition als Studium von Text und Sprache weiterhin treu bleibe. Doch würde man sich durch diese Argumentation den neuen Möglichkeiten verschließen, die sich dem Fach durch die stärkere Verzahnung mit anderen Wissensgebieten auftun. Warum sollte die Interpretation eines Romans nicht historische und soziale Zusammenhänge beleuchten, wenn sie in dem betreffenden Werk sichtbar werden? Soll sie sich wirklich damit begnügen, immer wieder die gleichen erzähltheoretischen Konzepte anzuwenden, die an anderen Romanen längst erschöpfend durchexerziert worden sind? Es gibt eben, dies scheint sich als Ergebnis der jahrelangen Grundsatzdebatte über das Thema Kulturwissenschaften herauszustellen, einen dritten Weg neben der Einschränkung und dem Verzicht auf literaturwissenschaftliche Kernkompetenzen. Entsprechend fordert Voßkamp von den Philologen »eine Öffnung und Erweiterung der Wissenschaft von Sprache und Literatur gegenüber den kulturwissenschaftlichen Nachbardisziplinen « (Voßkamp 2003: 73). Von einem Verzicht auf bewährte Arbeitsmethoden ist nicht die Rede; im Gegenteil. Mittelstraß stellte bereits 1987 fest: »Interdisziplinäre Kompetenz setzt disziplinäre Kompetenzen voraus« (Mittelstraß 1987: 154). 
Natürlich bleibt die Fremdsprachen-Didaktik von dieser Entwicklung, die die Geisteswissenschaften bis in ihre Grundfesten und universitären Strukturen erschüttert hat, nicht unberührt. Ganz im Gegenteil. Mit der Sprache, der damit eine viel umfassendere Funktion zukommt als nur diejenige des Kommunikationsmediums, wird auch die Vermittlung der Sprache aufgewertet. Wenn sich Kultur nur innerhalb semiotischer Zeichensysteme verwirklichen kann, diese Zeichensysteme aber zu einem untrennbaren Ganzen verwoben sind, öffnet der Sprachlehrer seinen Schülern gleichsam das Tor zu einer anderen Kultur. Durch dergleichen poetische Bilder mag er sich geschmeichelt fühlen, sollte sich aber zugleich bewusst werden, dass seine Verantwortung als interkultureller Mediator viel weitreichender ist als die des bloßen Vermittlers grammatisch-lexikalischer Kenntnisse. Diese Aufgabe verlangt von ihm klare Vorstellungen, wodurch die von ihm gelehrte Kultur eigentlich besteht und, vor allem, worin sie sich von der jeweiligen Kultur seiner Schüler unterscheidet. Hier könnte man erneut den Einwand erheben, in der Praxis seien Sprachkurse gerade im Rahmen der universitären Philologien dieser Mittlerfunktion schon immer nachgekommen, indem sie den Studierenden die Teilnahme an traditionellen literaturwissenschaftlichen, linguistischen und landeskundlichen Lehrveranstaltungen überhaupt erst ermöglichten. Vor dem Hintergrund der fächerübergreifenden Neuorientierung jedoch erweist sich ein stärkerer Austausch, wenn nicht gar ein VerflieBen der Sprachvermittlung mit den wissenschaftlichen Teildisziplinen als unbedingt notwendig.

An dieser Stelle kommt die Lyrik ins Spiel. Die Mentalität einer fremden Kultur, die Summe des Denkens und Empfindens einer Sprachgemeinschaft, lässt sich nirgends besser ablesen als in ihren Gedichten. Das expressive Potenzial, die Melodik und Rhythmik einer Sprache kommt nirgends so deutlich zum Vorschein wie in Texten, in denen jedes Wort absichtsvoll gewählt und sorgfältig auf sein Umfeld abgestimmt ist. In der Kombination von Pantomime und Vortrag sah ich daher eine Möglichkeit, brasilianische Deutsch-Lernende an authentische Phänomene deutschsprachiger Kultur heranzuführen, die sich von den artifiziellen und irgendwie sterilen Lehrwerktexten von Grund auf unterscheiden. Wer ein szenisches Bild zu einem Gedicht entwerfen möchte, muss sich detailliert und interessiert mit seinem Inhalt und seiner sprachlichen Charakteristik auseinandersetzen. Ich wollte, dass meine Studierenden die Verse sozusagen anfassten, damit sie sich ihrer phonetischen und semantischen Aspekte bewusst würden. (Die theoretische Grundlegung zu einer interkulturellen Literaturdidaktik liefert das Handbuch interkulturelle Germanistik [s. Wierlacher/Bogner 2003: 433-486]). Diese Methodik schien mir vorteilhaft, weil die bestehende Literaturdidaktik an der Deutschabteilung der UFRGS sehr »bodenständig « ist - mit allen positiven und negativen Konnotationen, die dieses Adjektiv hat: Auf solide, aber auch sehr konservative Weise lernen die Studierenden in drei Semestern die Epochen der deutschsprachigen Literaturgeschichte in chronologischer Abfolge anhand ausgewählter Texte kennen. Es bestand also Bedarf nach einem alternativen, progressiven Gegengewicht. Bei der Konzeption des Projektes ließ ich mich von grundsätzlichen Reflexionen Hubers leiten:

»[...] Gedichtchoreographien, wie sie im mutter- und fremdsprachlichen Unterricht vielfältig eingesetzt werden, [sind] nicht nur als andere Lesart neben der im Litera- 
turunterricht privilegierten analytischen $\mathrm{zu}$ verstehen, die den Studierenden neben dem kognitiven Zugang einen persönlich-affektiven, psychomotorischen Umgang mit dem literarischen Text und eine eigenständige produktive Textrezeption ermöglicht.« (Huber 2003: 340)

\subsection{Die Auswahl der Gedichte}

Warum ausgerechnet humoristische Gedichte? Erstens beabsichtigte ich, das erwähnte Vorurteil zu widerlegen, deutsche Literatur müsse notwendig ernst und »kopflastig « sein. Daher suchte ich bewusst nach Gedichten aus dem 19. und 20. Jahrhundert, um die kontinuierliche Traditionslinie des Komischen in der deutschsprachigen Lyrik zu belegen. Zweitens, sollte sich die Komik der Gedichte mit der Komik der Pantomimen zur Einheit fügen.

In der Vorbereitungsphase der eigentlichen Arbeit am Projekt gemeinsam mit den Studierenden suchte ich daher in zahlreichen Anthologien und Gedichtzyklen nach geeigneten Texten. Überflüssig zu bemerken, dass ich mein Augenmerk von vornherein auf die Werke von Autoren richtete, in denen ich am ehesten fündig zu werden hoffte. Dass die "Gebrauchslyrik « Erich Kästners oder Morgensterns »Galgenlieder « in diesem Fall ergiebiger als Klopstocks Oden sein dürften, ist gewiss spontan einleuchtend. Im Verlauf der Suche beeindruckte mich immer wieder, wie reich und vielfältig die Erscheinungsformen des Komischen in der Lyrik sind. Die Schilderung bizarrer Situationen, geistvoller Sprachwitz, paradoxe Gedankengänge, feine Ironie und derb-burleske Komik - all dies ist in den Gedichten vertreten. So hat Peter Rühmkorfs Kunststück, als bislang erster Lyriker deutscher Zunge einen Reim auf das Wort »Menschen« zu schaffen - »Die schönsten Verse der Menschen/[...] sind die Gottfried Bennschen « (zitiert nach Thalmayr 2004: 28) - einen völlig anderen
Charakter als Bert Brechts Gedicht von der Kellerassel (Tierverse, 4. Strophe):

Es war einmal eine Kellerassel

Die geriet in ein Schlamassel

Der Keller, in dem sie asselte

Brach eines schönen Tages ein

So daß das ganze Haus aus Stein

Ihr auf das Köpfchen prasselte.

Sie soll religiös geworden sein.

(Brecht [1934] 1967: 508-509)

Wieder anders verhält es sich mit Ernst Jandls rinks und lechts, die man angeblich "nicht velwechsern « könne - »werch ein Illtum!« (Jandl 1966: 175). Wer mitdenkt, begreift am Ende sogar, warum das Gedicht lichtung heißt, obwohl es gar keine kahle Stelle im Wald thematisiert.

Häufig wird Komik durch erzwungene Reime erzeugt; längst nicht nur bei Morgensterns oft zitiertem Wiesel, das ausgerechnet »auf einem Kiesel/inmitten Bachgeriesel« sitzen muss. In Dr. Heinrich Hoffmanns Struwwelpeter heißt es etwa: »Am Brunnen stand ein großer Hund/ Trank Wasser dort mit seinem Mund « (Hoffmann [1945] 1917: 4). Das Reimwort ist amüsant, weil es erstens "Schnauze" heißen müsste und überhaupt die ganze Modaladverbiale überflüssig wie ein Kropf ist: Schon klar, dass er nicht mit den Ohren trinkt! Dr. Hoffmann befindet sich in diesem Fall auf Augenhöhe mit Wilhelm Busch, der eine Bildergeschichte über einen Menschen namens Joseph, dem ausgerechnet an seinem Namenstag alles schief geht, mit folgendem Verspaar eröffnete: »Josephitag ist, wie du weißt, / Ein Fest für den, der Joseph heißt « (Busch 1960: II, 481). Ach ja?! Wie ungemein aufschlussreich...

Doch kommt Komik in Gedichten oft ganz anders zustande. Morgensterns Palmström wird, modern ausgedrückt, in der Fußgängerzone von einem Auto angefahren und kommt nach eingehender Prüfung des Vorfalls zu dem Ergebnis, dass er den Unfall nur geträumt habe - 
»Weil, so schließt er messerscharf, / Nicht sein kann, was nicht sein darf « (Morgenstern 1965: 263). Heinrich Heine, dieser Text ist mindestens genauso berühmt, sieht ein Fräulein am Meer in den Sonnenuntergang schmachten. "Sein Sie munter«, ruft er ihr zu, es sei ja doch immer dasselbe: »Hier vorne geht sie unter/und kehrt von hinten zurück« (zitiert nach Jacobs 2004: 79). Der Sprachwitz aus den Beispielen von Hoffmann und Busch blitzt hier nicht auf. Beide Gedichte, Morgensterns wie Heines, ziehen ihre Komik stattdessen aus der Schilderung einer Situation bzw. deren unerwarteter Bewertung. Der eine nimmt jedoch die übertriebene Gesetzesgläubigkeit der Deutschen satirisch aufs Korn ein Phänomen also, das in der Realität außerhalb der literarischen Texte existiert -, der andere stößt sich energisch von der romantischen Lyrik $a b$, die sich allzu einseitig als Ausdruck von Innerlichkeit versteht.

Der Lyriker Robert Gernhardt schrieb einen aus zehn Thesen zum komischen Gedicht bestehenden Feuilleton-Artikel, den die Frankfurter Allgemeine Zeitung am 12. Februar 2004 veröffentlichte. Das komische Gedicht brauche die Regel, lautet eine dieser Thesen, denn »Komik lebt von vorgegebenen Ordnungssystemen « (vgl. Gernhardt 2004), die sie durchkreuzt. Die Beispiele von Morgenstern und Heine zeigen, dass es sich ebenso um soziale wie um literarische Ordnungssysteme handeln kann.

Derlei Kritik an der Mentalität einer Epoche oder eines Volkes richtet ihren Fokus natürlich auf ein ausgesprochen abstraktes Objekt. Es geht auch ganz anders. Man denke etwa an die populäre Moritat aus der Mitte des 19. Jahrhunderts, die vom Attentat des preußischen Bürgermeisters Ludwig Tschech auf seinen König Friedrich Wilhelm IV. erzählt. Hier rückt ein konkretes historisches Ereignis ins Zentrum des Gedichts. Selbstverständlich geht es in der Handlung so makaber zu, wie es dieses lyrische Genre nun einmal erfordert. Nachdem wir den glimpflichen Ausgang des Mordversuchs erfahren haben - Tschech feuerte zwei Schüsse auf das Königspaar ab, doch wie durch ein Wunder blieben beide unverletzt -, ereifert sich der Bänkelsänger mit geheuchelter Empörung:

War wohl je ein Mensch so frech wie der Bürgermeister Tschech?

Der verruchte Übelthäter, Hochverräter, Attentäter!

Fast den König bracht er um vor dem ganzen Publikum, schoß sogar der Landesmutter durch den Rock ins Unterfutter. (zitiert nach Grünefeld 2006: 125)

Wieder ist es ein Reimpaar, das maßgeblich zur komischen Wirkung des Textes beiträgt. Mit dem hehren Wort »Landesmutter « begibt sich der Autor auf die höchste Stilebene, nur damit er im folgenden Vers zum trivialen Objekt Unterfutter hinabstürzen kann. Hinzu kommt, dass er den Schuss ins Unterfutter nicht als Glück im Unglück für das Opfer, sondern als Gipfel der Respektlosigkeit ausweist. Der Königsmord, so scheint der Text zudem anzudeuten, wäre halb so schlimm, wenn er diskret in den Gemächern seiner Majestät geschehen wäre aber vor Aller Augen! Der Sprechende äfft in diesem Fall die Stimmen der braven, spießbürgerlichen Untertanen nach, bringt aber keineswegs sein eigenes, persönliches Empfinden zum Ausdruck.

Damit ist ein wichtiges Stichwort genannt, das lange Zeit zu einer vielleicht nicht falschen, aber sicherlich unvollkommenen Lyrikdefinition beigetragen hat: Seit Herder, vor allem aber seit Goethe und Hegel behauptet sich die Vorstellung, dass Gedichte Innerlichkeit, also Stimmungen und Gefühle wiedergäben (vgl. Burdorf 1997: 4). Noch Emil Staiger 
erklärt Mitte des 20. Jahrhunderts, in der Lyrik werde die Grenze zwischen dem Menschen und der Natur aufgehoben, so dass sie miteinander zur Einheit verschmölzen (vgl. Staiger 1946: 47-54). Diese Kommunion kann nicht anders sein als ernst und feierlich. Keineswegs zielt sie darauf $a b$, den Leser zum Lachen zu bringen. Vor dem Hintergrund dieses Gattungsverständnisses nimmt sich der Begriff »komische Lyrik « geradezu als contradictio in adiecto aus. Die Erlebnisund Stimmungslyrik lädt uns ein, die Gefühle des Sprechenden empathisch mitzuempfinden. Humoristische Gedichte hingegen enthalten keinerlei Identifikationsangebot; stattdessen versuchen sie, uns das Widersinnige, Paradoxe und Verwerfliche ihrer Gegenstände vor $\mathrm{Au}$ gen zu führen, nehmen also eine distanzierte Haltung ein. Sie führen ihr Schattendasein in der Literaturgeschichte nicht zuletzt deshalb, weil ihnen in der Regel das vermeintliche Grundmerkmal des Gedichttextes fehlt: das lyrische Ich. Sofern sie sich nicht im reinen Sprachspiel erschöpfen, das im Extremfall völlig entsemantisiert sein kann, handeln sie meist in der dritten Person von "anderen«. Diese Gestalten - ganz gleich, ob Morgensterns Palmström, sein Mondkalb oder Ringelnatzens Kuddel Daddeldu sind der Gegenstand des Spottes, wobei sie in ihrer Schrulligkeit die Lacher durchaus auf ihrer Seite haben können. In jedem Fall wird sich der Leser von ihnen jedoch distanzieren, statt sich mit ihnen zu identifizieren. Falls doch einmal ein explizites Subjekt auf den Plan tritt, ist es meilenweit von Emil Staigers lyrischem Ich entfernt. Wir vernehmen die Stimme einer komischen Figur, die in extrem gespanntem Verhältnis zu den Normen ihres Umfeldes steht und uns dadurch zum Lachen reizt. Natürlich kann diese Heiterkeit die Form von Selbstironie annehmen, denn in den
Schwächen, die das Ich im Gedicht preisgibt, erkennen wir nicht selten unsere eigenen. Deutlich tritt die Selbstironie etwa in der Lyrik Robert Gernhardts zutage. Seine Verse eines Sterbenden, der dem Sensenmann in der Todesstunde mit lebenslang trainierter Höflichkeit begegnet, haben einen bitteren Beigeschmack:

Ach, noch in der letzten Stunde werde ich verbindlich sein. Klopft der Tod an meine Türe, rufe ich geschwind: Herein!

Woran soll es gehn? Ans Sterben?

Hab' ich zwar noch nie gemacht, doch wir werd'n das Kind schon schaukelnna, das wäre ja gelacht!

Interessant so eine Sanduhr! Ja, die halt ich gern mal fest. Ach - und das ist Ihre Sense? Und die gibt mir dann den Rest?

[...]

Ja, die Uhr ist abgelaufen.

Wollen Sie die jetzt zurück?

Gibt's die irgendwo zu kaufen?

Ein so ausgefall'nes Stück

Findet man nicht alle Tage, womit ich nur sagen will - ach! Ich soll hier nichts mehr sagen?

Geht in Ordnung! Bin schon

(Gernhardt 2006: 579-580)

Das Verhalten des lyrischen Ich dem Tod gegenüber scheint der Tragik der Situation völlig unangemessen, da es sich in banalem, prosaischem Geplauder erschöpft. Andererseits: weshalb sollte es dem Unvermeidbaren mit Tiefsinn oder Protest begegnen? An seinem endgültigen Verstummen änderte es doch nichts. In diesem Fall veranlasst uns das komische, von der Norm abweichende Verhalten des Ich nicht zu befreiendem Lachen, weil wir uns durchaus in ihm wiedererkennen. Früher oder später werden wir uns in derselben Lage befinden. Mir ist durchaus bewusst (auch wenn ich es bisher nicht explizit thematisiert habe), dass der Gattungsbegriff »komische Ly- 
rik « nicht unproblematisch ist. Auf keinen Fall lässt sich dieses Genre ex negativo als Negation der Erlebnislyrik innerhalb des gemeinsamen Rahmens der lyrischen Poesie definieren. Außer Gedichten mit komischer Wirkungsabsicht existiert eine breite Vielfalt anderer, die ebenso wenig erlebnishaft sind; man denke beispielsweise an Brechts Fragen eines lesenden Arbeiters oder manche Experimente der Konkreten Lyrik. Das Komische, ganz egal, in welcher Kunstform, lässt sich eben nicht auf textinterne Merkmale zurückführen, wenn auch unbestritten ist, dass es sich immer wieder derselben Stilmittel bedient. Es geht mir hier nicht darum, die unterschiedlichen Ansätze der Komiktheorie gegeneinander abzuwägen und auf die lyrische Textgattung anzuwenden. Zwar beschreibe ich einige rekurrente Stilmittel komischer Lyrik und erläutere die spezifische Komik einiger Gedichte. Gemeinsam mit Heiner Link, dem Herausgeber der Anthologie Die Laus im Uhrgehäuse. Komische Gedichte von Morgenstern bis Gernhardt (vgl. Link 2001), akzeptiere ich jedoch das Komische als Phänomen, das sich auf keine allgemeine Formel bringen lässt.

Stattdessen ist das Komische eine wirkungsästhetische Kategorie, die all jenen Texten zuzusprechen ist, die ihr Publikum zum Lachen reizen. Gernhardt definiert das komische Gedicht ebenfalls aus seiner Wirkungsabsicht heraus: Es ziele auf das Lachen seines Lesers oder Zuhörers ab. Allerdings, so fügt er hinzu, erschöpfe es sich nicht in dieser Zielsetzung. Für den humoristischen Lyriker sei der Weg zur Pointe ebenso wichtig wie die Pointe selbst (vgl. Gernhardt 2004).

Es ist daher von der subjektiven Wahrnehmung der Leser abhängig, die auf ein und denselben Text ganz unterschiedlich reagieren können. Gerade der prekäre Charakter des Komischen macht es jedoch für die kulturwissenschaftlich per- spektivierte Literaturbetrachtung interessant. Offenbar bestehen in Kulturgemeinschaften Übereinkünfte, was Heiterkeit auslöst und was nicht. Pointiert formuliert lautet daher die Frage, die das Projekt Fünf lyrische Pantomimen aufwerfen möchte: Worüber lachen Deutsche?

Ausgehend von diesen Grundgedanken schien es mir notwendig, Texte von ganz verschiedenen Autoren zu finden, die auf ganz verschiedene Weise komisch sind. Überdies kamen natürlich nur Gedichte in Frage, die sich aufgrund ihrer Inhalte zur szenischen Darstellung eigneten, was die Auswahl stark einengte. Denn bekanntlich enthalten lyrische Texte meist keine Handlung, die sich performativ nachspielen ließe. Selbst wenn sie komisch sind, neigen sie zur abstrakten Argumentation, wie das Beispiel von Wilhelm Buschs Lob der Selbstkritik unmissverständlich zeigt:

Die Selbstkritik hat viel für sich. Gesetzt den Fall, ich tadle mich: So hab ich erstens den Gewinn, $\mathrm{Daß}$ ich so hübsch bescheiden bin; Zum zweiten denken sich die Leut, Der Mann ist lauter Redlichkeit; Auch schnapp ich drittens diesen Bissen Vorweg den andern Kritiküssen; Und viertens hoff ich außerdem Auf Widerspruch, der mir genehm. So kommt es denn zuletzt heraus, $\mathrm{Daß}$ ich ein ganz famoses Haus.

(Busch 1960: II, 496)

Da wäre es noch leichter, Heines »Fräulein am Meere« gestisch-mimisch zu verkörpern, das herzergreifende Seufzen der Dame ließe sich gut überzeichnen. Meine Wahl fiel jedoch auf zwei andere Texte von Heine, nämlich Ein Jüngling liebt ein Mädchen, berühmt vor allem durch Robert Schumanns kongeniale Vertonung. Wilhelm Busch schrieb für mein Vorhaben ungleich geeignetere Gedichte. Ich entschied mich schließlich für eine szenische Bearbeitung der Tierfabel Die Teilung. Hinzu kamen Christian Morgen- 
sterns Vice-Versa, Heinz Erhardts Der Einsame und Ernst Jandls calypso. Im Folgenden gehe ich auf die Gründe für meine Entscheidungen und auf die szenischen Darstellungen der fünf Gedichte ein.

\section{Die Projektarbeit mit den Studieren- den}

\subsection{Die Entwicklung szenischer Bilder zu den Gedichten}

Im Zentrum der Projektarbeit standen natürlich nicht die bislang vorgestellten Grundgedanken des Sprachdidaktikers und Literaturwissenschaftlers. Stattdessen machten, nach Vorgesprächen mit Gruppen von Studierenden, sieben über vier Wochen verteilte, etwa dreistündige Proben den Löwenanteil der Arbeit aus. Dabei war nicht von vornherein abzusehen, zu welchem Ende das Projekt führen würde. Fest stand nur, dass das Ergebnis am 16. Mai dem Publikum der Faculdade de Letras präsentiert werden sollte, man also stringent und zielstrebig würde vorgehen müssen. Sicher: Eine ungefähre Vorstellung, welche Bilder man zu welchen Gedichten würde entwickeln können, hatte ich im Vorhinein schon; sonst hätte ich die fraglichen Texte vermutlich nicht ausgewählt. Aber in der Praxis und im Detail kam meist alles ganz anders; so im Fall des zuerst einstudierten Bildes, der pantomimischen Darstellung von Heines Ein Jüngling liebt ein Mädchen. Gemäß dem chronologischen Prinzip eines Arbeitsberichts schildere ich die Entstehung der fünf Szenen in ihrer zeitlichen Reihenfolge, bevor ich auf ihre endgültige »Montage« zu sprechen komme. Die Gedichte sind um ihre portugiesischen Übersetzungen ergänzt, die ich gemeinsam mit einem Studenten anfertigte, um sie ins Libretto unseres Stücks aufzunehmen.

\section{Bild}

\section{Heinrich Heine}

\section{Ein Jüngling liebt ein Mädchen}

Ein Jüngling liebt ein Mädchen,

Die hat einen andern erwählt;

Der andre liebt eine andre,

Und hat sich mit dieser vermählt.

Das Mädchen heiratet aus Ärger

Den ersten besten Mann,

Der ihr in den Weg gelaufen;

Der Jüngling ist übel dran.

Es ist eine alte Geschichte,

Doch bleibt sie immer neu;

Und wem sie just passieret,

Dem bricht das Herz entzwei.

(Heine [1827] 1975: I/I, 171)

\section{Um Rapaz Ama Uma Moça}

Um rapaz ama uma moça,

Esta escolheu um outro;

Este ama uma outra

E casa-se com esta.

A moça, de raiva, casa-se

Com o primeiro homem

Que cruza o seu caminho -

Com isso, o rapaz sofre.

É uma velha história,

Que, no entanto, ainda se mantém;

E tão-só aquele que por isso passa

Sabe como um coração se despedaça.
Das Gedicht ist knapp, schlicht und unprätentiös. Gerade deswegen wirkt das Pathos, das es erst in der allerletzten Zeile herausbildet, trotz der verbrauchten Metapher vom gebrochenen Herzen überzeugend. Der Text vermittelt ein Gesche- hen, enthält aber keine fiktionale Handlung im eigentlichen Sinne. Denn der Jüngling und alle anderen Beteiligten dieser zum Fünfeck erweiterten Dreiecksgeschichte sind keine erfundenen Personen mit individuellen Merkmalen, 
wie es die Figuren in einem Roman für gewöhnlich sind. Stattdessen haben wir es mit der abstrakten Idee einer zwischenmenschlichen Situation zu tun, die sich alle Tage nach demselben Schema ereignet und gar nichts Besonderes ist. Für den betroffenen Jüngling (der natürlich genauso gut weiblich sein kann!) ist sie dennoch ausgesprochen leidvoll. In diesem Zusammenfall des Tragischen mit dem Alltäglichen liegt die bittere Komik des Gedichts.

Heine zeigt uns die Entwicklung dieser Situation, als wäre sie eine Figurenkonstellation auf dem Schachbrett. Deswegen vermutlich kamen wir spontan auf den Gedanken, fünf Akteure, natürlich drei Männer und zwei Frauen, auf der leeren Bühne zu positionieren. Die Rezitatorin des Gedichts stand abseits im Vordergrund, präsent, aber nicht unmittelbar am Spiel beteiligt. Was die Pantomimen aufführten, sobald sie mit ihrem Vortrag einsetzte, glich einer Kettenreaktion, die sich fließend von rechts nach links fortsetzte: Der Jüngling, ganz außen rechts, versucht, seine Nebenfrau an sich zu ziehen, diese stößt ihn missbilligend von sich, wendet sich schmachtend ihrem anderen Nachbarn zu, erntet aber ihrerseits einen verächtlichen Blick des Mannes auf der zentralen Position, der vor seiner anderen, beglückten Nebenfrau das Knie beugt und ihr einen Ring an den Finger steckt - laut Heine vermählt er sich ja mit ihr. Nichts Böses ahnend, schlendert von links außen der »erste beste Mann « ${ }^{1}$ nach der anderen Bühnenseite, wo er vom Schwarm des Jünglings energisch gepackt wird. Damit ist das Ende der zweiten Strophe erreicht. Der Jüngling, eigentlich im Fokus des Gedichts, tritt nach vorn an die Rampe. "Dem bricht das Herz entzwei«: Er zieht ein rotes Herz aus Pappkarton aus der Innentasche des Jacketts, reißt es mittendurch und lässt die beiden Hälften zu Boden fallen.

Am meisten erstaunte mich dabei, wie selbstverständlich die Studierenden in die reduzierte, zeichenhafte Gestik hineinfanden. Niemand verfiel auf den Gedanken, man müsste natürlicher spielen, das Geschehen tendenziell so darstellen, wie wir es im wirklichen Leben erfahren. Offenbar erkannten alle intuitiv, dass das Ausformen der Figuren zu Charakteren mit konkreten Eigenschaften dem universellen Gedichtinhalt nicht gerecht würde. ${ }^{2}$ Die Kürze des Textes hätte dies nicht zwingend verhindert, weil der Vortrag problemlos ausgesetzt werden kann, während die Pantomimen weiteragieren. Im Hinblick auf die Abstraktheit bildete Ein Jüngling liebt ein Mädchen das entgegengesetzte Extrem zu Buschs Die Teilung. Doch erst einmal Morgenstern (s. 2. Bild).

Kennzeichnete schon Heines Text das lyriktypische Merkmal der relativen Kürze, so ist Morgensterns Vice Versa noch konzentrierter und verdichteter. Acht Verse,

1 Diese Formulierung Heines führt uns vor Augen, dass es nicht immer das gesuchte Vokabular ist, das Fremdsprachler vor Verständnisprobleme stellt. Es war nicht einfach, meinen Studierenden zu erklären, dass »der erste beste Mann« gerade nicht »der erstbeste Mann« ist.

2 Huber formuliert allgemein, worauf es bei der Adaption des Gedichts als lyrische Pantomime ankommt: »[...] In erster Linie gilt es, die Teilnehmer für den Medienwechsel zu sensibilisieren, der in den handlungs- und produktionsorientierten Verfahren stattfindet: Es ist der Sprung vom zweidimensionalen, stummen, unbewegten, überzeitlichen, visuell rezipierten und den Rezipienten vereinzelnden Medium der Schrift in den dreidimensionalen Raum der von Körper und Stimme getragenen, visuell-auditivkinästhetisch und im Publikumskollektiv rezipierten, bewegten und ephemeren Zeichen der Zeitkünste Tanz und Theater.« (Huber 2003: 340) 


\section{Bild}

\section{Christian Morgenstern}

\section{Vice Versa}

Ein Hase sitzt auf einer Wiese, des Glaubens, niemand sähe diese.

Doch, im Besitze eines Zeißes, betrachtet voll gehaltnen Fleißes vom vis-à-vis gelegnen Berg ein Mensch den kleinen Löffelzwerg. Ihn aber blickt hinwiederum in Gott von fern an, mild und stumm.

\section{Vice-versa}

Uma lebre está sentada num prado, Acreditando que ninguém a vê.

Porém, em posse de um binóculo,

Um homem observa, com redobrado esforço,

Situado no morro mais próximo,

A pequenina de orelhas compridas.

Mas ele, por sua vez, também é observado:

de longe, fita-o um deus - brando e mudo.

(Morgenstern 1965: 280)

die auf vier Strophen entfallen, wobei der erste und der letzte Zweizeiler den vierzeiligen Satz in der Mitte symmetrisch umrahmen. Wenn man den Inhalt in Beziehung zur Form setzt, kann man unschwer erkennen, dass perfekte Übereinstimmung besteht. Die drei Sätze thematisieren jeweils eine der drei »Personen « des Gedichts, den Hasen, den Menschen und den Gott, wobei sich die Strophen 2 und 3 auf das unmittelbar zuvor genannte Wesen zurückbeziehen.

Die Weltordnung in einer Streichholzschachtel - man sollte denken, die Figuren blieben hier genauso unbestimmt wie bei Heine. Doch das Gegenteil ist der Fall. Wie ein detailverliebter Modelleisenbahner versieht Morgenstern alle drei Miniaturen mit individuellen Charakterzügen, worauf er ein Mindestmaß an Silben und Worten verwendet. Der Hase, der sich unbeobachtet glaubt, ist arglos; der Mensch, der ihn »voll gehaltnen Fleißes« ansieht, geduldig; der Gott, dies steht explizit im Text, »mild und stumm «. Sie alle werden vom Dichter, der sie gleichsam als vierte und höchste Instanz $\mathrm{zu}$ betrachten scheint, bei ihrem ernsten und sinnlosen Tun ironisch belächelt. Die Komik des Gedichts liegt in der Tatsache, dass die Wesen ihre Situation verkennen, sich also inadäquat zu ihrem Umfeld verhalten.

Wie sah die szenische Adaptation aus? Mit dem totalen Minimalismus der leeren Bühne ließ sich dieses Mal nicht arbeiten. Der Mensch benötigt schließlich einen Berg, der Gott muss von weiter oben auf ihn hinabblicken können. Die spontane Improvisation in der Probe ergab die endgültigen Lösungen für die Aufführung: ein Tisch mit einem Hocker darauf in der Bühnenmitte, eine hölzerne Staffelei voller Farbflecken in der linken hinteren Ecke. Anders als bei Heine, wo die Pantomime dem Gedichtinhalt eigentlich nichts hinzufügte, erhielt diese Szene, ausgehend vom Text, eigenwillige Züge. Wir flankierten die Bühne mit zwei epischen Elementen, auf der rechten Seite der Rezitator, auf der linken ein Musiker mit Violine und Blockflöte. ${ }^{1}$ Als erstes tritt der Hase auf, hoppelt ein wenig im Kreis herum und lässt sich schließlich vorne rechts nieder, auf diagonaler Achse

1 Das Wort »episch« ist hier im Brechtschen Sinne verwendet: Musiker und Rezitator treten als vermittelnde Elemente zwischen das szenische Spiel und die Zuschauer, ähnlich dem Erzähler im narrativen Text. 
mit der Staffelei und dem Berg. Begleitet wird er von Häschen in der Grube, mit den Fingerkuppen auf den Geigensaiten gezupft. Die Musik verstummt, der Rezitator trägt die erste Strophe vor. Sofort danach kommt mit maßlos übertriebenen Riesenschritten ein Mann hereingestapft, der, wie der »wilde Jägersmann« aus dem Struwwelpeter, ein überlanges Gewehr über der Schulter trägt (eigentlich war es ein zusammengeklapptes Stativ, das ich für Info-Veranstaltungen des DAAD benutze). Musikalisch untermalte diesen Auftritt die Blockflöte mit Ein J̈̈ger aus Kurpfalz..$^{1}$ »Der Mensch « stellt sich hinter seinem Berg auf und erspäht den Hasen durch eine Röhre aus Pappkarton, sein Fernglas der Marke Zeiss. In der Zwischenzeit spricht der Rezitator die zweite und auch gleich die dritte Strophe des Gedichts. Mit der Hand weist er nach der Staffelei, die jetzt ein weiterer Akteur besteigt, ganz in eine Toga aus weißem Leintuch gehüllt - er ist ja nicht der Gott nach monotheistischer Vorstellung, sondern ein, d.h. ein heidnischer Gott. Als Motiv, das dieses Mal auf der Violine gestrichen wird, assoziierten wir dennoch Halleluja. Von oben blickt er gütig auf den Jäger herab, der jetzt hinter seinem Berg hervorschleicht, die Flinte in Anschlag bringt und - indem ein Mitwirkender von außen in die Hände klatscht das Häschen abknallt.

Es ist offensichtlich, dass diese Szene insbesondere den Darstellern des Hasen und des Jägers eine starke pantomimische Leistung abforderte. Überhaupt kam sie nur in dieser Form zustande, weil sie zwei Studierende mit bemerkenswertem komödiantischem Talent ausgestalteten. Hinsichtlich der Requisiten be- schränkten wir uns auf dasselbe Minimum, mit dem sich Morgenstern auf sprachlicher Ebene zufriedengibt. Zweifellos wäre es kein Problem gewesen, dem Jäger einen echten Feldstecher in die Hand zu geben, zur Not hätten wir ein Jagdgewehr auftreiben können - aber das gewollt Provisorische und Verfremdete schien uns einfach reizvoller. Wozu ein echtes Fernglas, wenn es am Ende doch absurd bleibt, dass der »Mensch « damit aus dem Abstand von zwei Metern auf die Hasendarstellerin blickt? Jede Art von Illusionstheater, das dem Bühnengeschehen den Anschein des Wirklichen geben will, wäre in diesem Fall die falsche Lösung gewesen. Stattdessen erkannten wir im grotesk überzeichnenden Spiel die Möglichkeit, die ironische Distanz des Dichters zum Gegenstand seines Textes szenisch wiederzugeben. Dazu gehörte auch der makabere Schluss, den wir auf eigene Faust dazu erfanden.

Die Teilung von Wilhelm Busch (s. 3. Bild) unterscheidet sich von den ersten beiden Gedichten zunächst einmal durch seinen ungleich größeren Umfang. Lyrischknapp bleibt es aber dennoch, wenn man bedenkt, dass es innerhalb der fünf sechszeiligen Strophen eine relativ ereignisreiche Geschichte erzählt. Nirgends hält sich Wilhelm Busch mit Details auf, die nicht unmittelbar dazu beitragen würden, das fiktive Geschehen zügig seinem Ende zuzuführen. Gleich in den ersten beiden Versen ordnet er sein Gedicht in die Erzählgattung der volkstümlichen Tierfabel ein. Mit der beiläufigen Parenthese »so wird gesagt « bedeutet er uns augenzwinkernd, dass die folgende Geschichte nicht seine eigene Schöpfung sei, sondern einer uralten mündlichen Über-

1 Die Titel der Volkslieder waren im Programmheft nebst portugiesischer Übersetzung angeführt. Interessant ist, dass die Melodie von Häschen in der Grube in Brasilien jedermann bekannt ist, allerdings mit einem inhaltlich völlig verschiedenen Text gesungen wird. 


\section{Wilhelm Busch}

\section{Wilhelm Busch}

\section{Die Teilung}

Es hat einmal, so wird gesagt,

Der Löwe mit dem Wolf gejagt.

Da haben sie vereint erlegt

Ein Wildschwein, stark und gut gepflegt.

Doch als es ans Verteilen ging,

Dünkt das dem Wolf ein mißlich Ding.

Der Löwe sprach: »Was grübelst du?

Glaubst du, es geht nicht redlich zu?

Dort kommt der Fuchs, er mag entscheiden

Was jedem zukommt von uns beiden «. »Gut«, sagt der Wolf, dem solch ein Freund

Als Richter gar nicht übel scheint.

Der Löwe winkt dem Fuchs sogleich:

»Herr Doktor, das ist was für Euch.

Hier dieses jüngst erlegte Schwein,

Bedenkt es wohl, ist mein und sein.

Ich faßt' es vorn, er griff es hinten;

Jetzt teilt es uns, doch ohne Finten«.

Der Fuchs war ein Jurist von Fach.

"Sehr einfach «, spricht er, »liegt die Sach:

Das Vorderteil, ob viel, ob wenig,

Erhält mit Fug und Recht der König.

Dir aber, Vetter Isegrim,

Gebürt das Hinterteil. Da nimm!«

Bei diesem Wort trennt er genau

Das Schwänzlein hinten von der Sau.

Indes der Wolf verschmäht die Beute,

Verneigt sich kurz und geht beiseite.

»Fuchs «, sprach der Löwe, »bleibt bei mir,

Von heut an seid Ihr Großwesir«.

(Busch 1960: IV, 283/284)

lieferung entstamme. Als erfahrene Leser erkennen wir natürlich die Fiktionsironie dieser Behauptung und goutieren sie als Vorgeschmack auf den Sarkasmus des Folgenden.

»Nimmt man es buchstäblich, dann sollte jedes komische Gedicht zum ersten eine heitere Sprache sprechen, es sollte zweitens zugleich über etwas Belustigendes berichten«. (vgl. Hadulla 2005)

\section{A Partilha}

Diz-se que, certa vez,

O leão foi à caça com o lobo.

Juntos, caçaram um javali,

Gordo e suculento.

Porém, quando chegou a hora de dividir,

O lobo pressentiu algo errado.

Disse o leão: »O que estás a matutar?

Crês que estou a enganar-te?

Lá vem a raposa; que ela decida

O que cabe a cada parte «.

»De acordo«, diz o lobo, a quem não é nada mal

Ter como juiz uma amiga como tal.

De imediato, o leão acena para a raposa.

»Doutora, esta é para vós:

Temos aqui este javali recém abatido,

Vede bem, pertence a mim e a ele.

Eu peguei na frente, ele agarrou atrás;

Agora, dividi-o entre nós, mas sem trapacear «.

A raposa era uma jurista de profissão.

»Muito simples «, diz ela, »apresenta-se o caso:

A parte anterior, seja muito, seja pouco,

Cabe ao Rei, com razão e por direito.

Quanto a ti, meu caro Isegrim,

Resta a posterior. Leva-a e dá-te por satisfeito! «

Assim resolvendo, ela corta exatamente,

De trás do javali, o rabinho, tão-somente.

O lobo, porém, rejeita a presa,

Faz uma mesura e sai de cena.

»Raposa«, disse o leão, »ficai com o rei,

A partir de hoje, primeira-ministra sois por lei! «

Dem möchte ich nicht vorbehaltlos zustimmen, da mir eine mehr oder minder entsemantisierte Sprachkomik, die also rein gar nichts »berichtet", durchaus möglich scheint. Buschs Gedicht Die Teilung dürfte Hadullas Ideal aber zweifellos entsprechen.

Der traditionellen Fabel entspricht auch der bestimmte Artikel vor den Bezeich- 
nungen der Tiere: der Löwe und der Wolf, was eigentlich widersinnig ist, weil es mehr als nur ein Exemplar der jeweiligen Spezies gibt. Doch wie immer sind die Tiere abstrakte Prinzipien menschlichen Handelns. Der Löwe repräsentiert die Selbstherrlichkeit, der Wolf die Habgier, der Fuchs die Arglist. Klar, dass am Ende der Gefräßige das Nachsehen hat, während der Schlaue auf seine Kosten absahnt. Nicht von ungefähr spricht Buschs König Löwe dem dienstfertigen Fuchs das höchste politische Amt im Tierreich zu. Die implizite Sozialkritik wurde von den Studierenden hier in Brasilien, wo sich der politische Diskurs zum großen Teil um Korruption und Nepotismus dreht, im Fluge erfasst.

Aufgrund seines narrativen Charakters bietet sich dieses Gedicht viel eher zur szenischen Bearbeitung an als die beiden bereits besprochenen. Wir brauchten uns nicht den Kopf darüber zu zerbrechen, wie sich »lieben « oder »heiraten « gestisch darstellen ließen oder wie wir mit äußerst beschränkten Mitteln einen Berg auf die Bühne stellen könnten. Hier ging die konkrete Handlung des Gedichts ganz natürlich in szenisches Spiel über, das erneut beträchtliches Eigenleben entwickelte. Wilhelm Busch, das dürfte jedem Leser seiner Bildergeschichten bekannt sein, war bestimmt kein Gegner der Gewaltdarstellung. In diesem Fall jedoch schildert er nicht, wie Löwe und Wolf das Wildschwein reißen, weil sein Interesse ganz auf den nachfolgenden juristischen Teilungsakt gerichtet ist. Wir dagegen sahen gerade in diesem Geschehen die Möglichkeit, eines der dramatischen Highlights unseres Stückes zu setzen. Einer der Studenten bewies enormes Talent $\mathrm{zu}$ grunzen und zu quieken. $\mathrm{Zu}$ Beginn läuft er auf allen Vieren auf die Bühne, am Hosenboden eine Spirale aus Pappkarton befestigt: der Ringelschwanz. Die Wildsau freut sich des Lebens, grunzt zufrieden, quiekt vergnügt, während sich von hinten die Jäger anschleichen, in der Hand die Röhre, die Morgensterns »Menschen« als Fernrohr diente, bzw. einen geschlossenen Regenschirm, der später nochmals verwendet wird. Gebrüll und Gequieke, dann liegt die Sau erledigt am Boden. Erst jetzt betritt der Rezitator den Bühnenraum. Er beginnt mit seinem Vortrag, wird aber vom Tumult der »Tiere« unterbrochen, weil das Wildschwein noch einmal zum Leben erwacht. Ein missbilligender Blick auf die Akteure, dann fängt er von vorne an und liest das Gedicht bis zu Ende.

Die Pantomimen konnten das Tempo des Vorspiels nach Belieben variieren, während des Vortrags jedoch waren ihre Handlungen genau an den Wortlaut des Textes gebunden. Spricht der Löwe »Dort kommt der Fuchs..." , so muss der betreffende Darsteller bereits auf der Bühne stehen, damit der Löwe auf ihn deuten kann. Schwierig wird die Simultaneität von Erzählung und Spiel im Übergang von der vierten zur fünften Strophe. »Da nimm «! sagt der Fuchs, erst in den nachfolgenden Versen erfährt der Leser, was er im selben Augenblick tut. Wir entschieden uns, das Geschehen vorweg zu nehmen: Nach »...gebührt das Hinterteil« setzt der Rezitator aus. Der Fuchs in unserem Fall eine Füchsin, was mit dem femininen Substantiv raposa im Portugiesischen gut kombiniert - zieht ein riesiges Messer aus seinem Stiefel, kappt den Ringelschwanz und wirft ihn mit verächtlicher Gebärde dem ungläubig glotzenden Wolf hin. Erst bei dieser letzten Geste wird das »Da nimm«! gesprochen. Am Schluss beugt die Füchsin vor dem Löwen das Knie und erhält den Ritterschlag mit dem Regenschirm.

Der Komiker Heinz Erhardt, 1979 verstorben, war eine feste Größe der deutschen Fernsehunterhaltung und dürfte selbst Deutschen der jüngeren Genera- 


\section{Bild}

\section{Der Einsame}

Einsam irr' ich durch die Gassen, durch den Regen, durch die Nacht. Warum hast du mich verlassen? Warum hast du das gemacht?

Nichts bleibt mir, als mich zu grämen, gestern sprang ich in den Bach. Um das Leben mir zu nehmen, doch der Bach war viel zu flach.

Einsam irr' ich durch den Regen, und ganz feucht ist mein Gesicht. Nicht allein des Regens wegen, nein, davon alleine nicht.

Wo bleibt Tod im schwarzen Kleide? Wo bleibt Tod und tötet mich? Oder besser noch: Uns beide. Oder besser: Erst mal dich!

(Erhardt 1970: 201)

tion ein Begriff sein. Im Ausland hingegen kennt ihn kaum jemand. Besser so, werden manche Deutsche spontan sagen, womit sie dem Künstler bitter unrecht tun. Denn Erhardt war ein vielseitig begabter Entertainer, der seine Kapazitäten in der Filmrolle des Willi Kuckuck beileibe nicht erschöpfte. Während er auf der Kinoleinwand das Verlangen des $\mathrm{Pu}-$ blikums nach Klamauk bediente, schuf er ein umfangreiches lyrisches Werk, das durch eine ungleich feinsinnigere Komik besticht. Als gelungenes Beispiel poetischer Selbstreflexion zitiere ich hier das folgende Gedicht mit dem Titel Beichte:

»Warum machst du in Gedichten«? fragte mich ein Menschenkind.

»Warum schreibst du nicht Geschichten, die doch leicht verkäuflich sind «?

Oh, ich habe meine Gründe für mein Tun - und sprach verträumt: »Weil ich es viel schöner finde, wenn sich hinten alles reimt." (Erhardt 2008: 94)

So interessant dergleichen humoristisch verpackte Gedanken zur literarischen
Ästhetik sind, in Pantomimen lassen sie sich schwerlich umwandeln. Der Einsame bietet sich zur szenischen Adaptation vielleicht nicht auf den ersten Blick an, lässt sich aufgrund seiner Schlusspointe jedoch als eine Art Sketch wiedergeben.

Erneut kam unser Musiker zum Einsatz: Während er auf der Flöte das melancholische Intermezzo sinfonico aus Mascagnis Cavalleria rusticana spielte, drehte eine Akteurin mit aufgespanntem Regenschirm auf der Bühne ihre Runden. Nachdem die Musik verklungen ist, spricht sie Erhardts Text, teilweise an einen Mann gewandt, der desenvolviert rauchend in einer der vorderen Bühnenecken steht. Er ist natürlich das nur in ihrer Phantasie anwesende Du, von dem sie verlassen worden ist. Text und Vortrag leben vom falschen Pathos, dessen Scheinheiligkeit erst ganz am Ende offenbar wird. Doch lässt Erhardt die mangelnde Authentizität des Gefühls schon vorher durchblitzen, insbesondere durch den Sprung in den seichten Bach, der das Fehlen echter Selbstmordabsichten ent- 
larvt. Dann die letzte Strophe: Von hinten nähert sich der beschworene »Tod im schwarzen Kleide", den die Rezitatorin erst bemerkt, als er mit weit aufgerissenem Maul und ausgefahrenen Klauen nach ihr greifen will. »Oder besser: Erst mal dich!«: Da der Tod in Wahrheit doch ein wenig schrecklicher ist, als sie es sich in ihrem Liebeskummer vorgestellt hat, bietet sie ihm den Verflossenen als Beute an, der seinerseits nicht interessiert ist: $\mathrm{Er}$ stürzt hinaus, der Tod hinterher.

5. Bild

\begin{tabular}{|c|c|c|}
\hline \multicolumn{3}{|c|}{ Ernst Jandl } \\
\hline calypso & & \\
\hline $\begin{array}{l}\text { ich was not yet } \\
\text { in brasilien } \\
\text { nach brasilien } \\
\text { wulld ich laik du go }\end{array}$ & $\begin{array}{l}\text { als ich anderschdehn } \\
\text { mange lanquidsch } \\
\text { will ich anderschdehn } \\
\text { auch lanquidsch in rioo }\end{array}$ & $\begin{array}{l}\text { yes yes de senden } \\
\text { mi across de meer } \\
\text { wer ich was not yet } \\
\text { ich laik du go sehr }\end{array}$ \\
\hline $\begin{array}{l}\text { wer de wimen } \\
\text { arr so ander } \\
\text { so quaitt ander } \\
\text { denn anderwo }\end{array}$ & $\begin{array}{l}\text { ich was not yet } \\
\text { in brasilien } \\
\text { nach brasilien } \\
\text { wulld ich laik du go }\end{array}$ & $\begin{array}{l}\text { ich was not yet } \\
\text { in brasilien } \\
\text { nach brasilien } \\
\text { wulld ich laik du go }\end{array}$ \\
\hline $\begin{array}{l}\text { ich was not yet } \\
\text { in brasilien } \\
\text { nach brasilien } \\
\text { wulld ich laik du go }\end{array}$ & $\begin{array}{l}\text { wenn de senden } \\
\text { mi across de meer } \\
\text { wai mi not senden wer } \\
\text { ich wulld laik du go }\end{array}$ & \\
\hline
\end{tabular}

So viel dürfte vermutlich jedem einleuchten: Dieses Gedicht ist, wie der größte Teil des poetischen Werkes Ernst Jandls, völlig unübersetzbar. Jeglicher Versuch, die bizarre Mischung aus Deutsch und Englisch in eine vergleichbar bizarre Mischung aus Portugiesisch und Englisch umzuwandeln, hätte sich allzu weit vom Original entfernt. Bei der Erstellung des Programmheftes ließen wir daher die Finger davon.

Es bedarf keiner Erklärung, dass ich diesen Text auswählte, weil es sich um ein Brasiliengedicht handelt. Doch ist es wirklich ein Brasiliengedicht? Eigentlich spricht das lyrische Ich nicht vom größten südamerikanischen Land mit seinem enormen natürlichen und kulturellen Reichtum; es drückt vielmehr seine stereotypen Vorstellungen vom mutmaßlichen Tropenparadies aus. Darauf nimmt auch der Titel calypso Bezug, der neben einer Nymphe aus der grie- chischen Mythologie auch einen Musikstil aus der Karibik bezeichnet. Zwar liegt Brasilien nicht wirklich in der Karibik, aber was kümmert das einen mitteleuropäischen Touristen oder Geschäftsreisenden, der in der Hauptsache an "quaitt anderen wimen " interessiert ist? Jandls Gedicht erzeugt seine Komik einerseits durch den ignoranten Blick auf das fremde Land, andererseits durch den grotesken Mischmasch zweier Sprachen. Dass es mit der Selbsterkenntnis des Gedichtsubjektes nicht weit her ist, geht aus der vierten Strophe hervor: Obwohl sein Englisch ziemlich deutsch klingt, hält es sich für polyglott und sprachbegabt.

Die ironische Brechung des Textes ist deutlich akzentuiert, wird aber dennoch nicht von allen Lesern und Interpreten wahrgenommen. Der brasilianischen Germanistin Celeste Ribeiro de Souza etwa scheint die Zeichnung des lyrischen Ich als lächerliche Figur völlig verborgen 
zu bleiben. In ihrer Monographie Retratos do Brasil, das Brasilienbilder in der deutschsprachigen Literatur untersucht, widmet sie Jandls Gedicht eine halbseitige Analyse. Das Ich drücke sein Verlangen nach den exotischen Frauen Brasiliens aus, heißt es als abschließendes Resümee (vgl. Ribeiro de Souza 1996: 186187). Stimmt schon. Aber dahinter steckt eine satirische Sprechabsicht, die dem Gedicht erst seine eigentliche Bedeutung gibt.

Wir hielten es für geboten, unsere $\mathrm{Zu}$ schauer mit entsprechenden Hinweisen in die richtige Richtung zu lenken. Ein deutscher Austauschstudent übernahm den Part des Rezitators, der als zentrales Ich des Gedichts unmittelbar am Spiel beteiligt ist. Sein Auftritt erhielt kabarettistische Züge, indem er als Tourist mit Kamera, Bermudas, Sonnenhut und -brille auf die Bühne kam. Als erstes drückte er einem Zuschauer in der ersten Reihe seinen Fotoapparat in die Hand, damit er ihn vor Rio de Janeiros Cristo Redentor ablichte, dargestellt von einer Akteurin, die hinter ihm mit ausgebreiteten Armen auf einem Stuhl stand. Der Vortrag des Gedichts war von assoziativen Symbolen begleitet. Annähernd mimetisches Spiel, wie im Fall von Buschs Tierfabel, kommt hier nicht in Frage, denn Jandls Text erzählt keine Geschichte. So ließen wir während der zweiten Strophe eine Mitwirkende $\mathrm{zu}$ den Klängen des Girl from Ipanema im SambaSchritt über die Bühne tanzen, unser Rezitator starrte ihr mit offenem Mund hinterher. Zwei weitere Akteure trugen auf der flachen Hand ein Papierschiff sowie, scheinbar unter größter Anstrengung, einen Stapel portugiesischer Wörterbücher durch das Bild, als von der Reise »across de meer « und der "lanquidsch in rioo" die Rede ist. Immer dann, wenn der Rezitator »Brasilien « sagte, also insgesamt acht Mal, hielt eine Studentin ganz me- chanisch eine brasilianische Flagge hoch. Die experimentelle Sprache musste sich im experimentellen Spiel widerspiegeln.

\subsection{Struktur und Rahmen des Stückes}

Schon während der Entwicklung der fünf Bilder diskutierten wir darüber, in welcher Reihenfolge sie aufgeführt werden sollten. Dabei wurden ganz unterschiedliche Kriterien in Betracht gezogen. So schien es beispielsweise unvorteilhaft, ausgerechnet das avantgardistische Jandl-Gedicht an den Anfang zu stellen, weil wir unser Publikum langsam an das Prinzip der »lyrischen Pantomimen « gewöhnen wollten. Folglich kamen wir frühzeitig überein, dass wir den $\mathrm{Zu}$ schauern calypso erst ganz zuletzt, gleichsam als Dessert, servieren würden. Hinzu kam, dass wir eine Möglichkeit erkannten, die beiden Texte zum Thema Liebe, also Heine und Erhardt, miteinander zu verbinden: Eine der enttäuschten Personen aus Ein Jüngling liebt ein Mädchen, d. h. eine der Titelfiguren, die beide ihr Liebesglück nicht finden, könnte als Erhardts Einsame(r) weiterspielen. Tatsächlich übernahm die Darstellerin des »Mädchens « die Hauptrolle im folgenden Bild. Während die übrigen Akteure der Pantomime auf Heines Gedicht die Bühne verließen, spannte sie ihren Regenschirm auf, um einsam durch die nächtlichen Gassen zu irren.

Doch blieb diese Art von Verknüpfung die Ausnahme und sollte es auch bleiben. Im Allgemeinen wollten wir den fragmentarischen, zuweilen improvisiert wirkenden Charakter unseres Stückes beibehalten. Das kurze Morgenstern-Gedicht mit den drei musikalischen Einlagen bot sich als Ouvertüre an, darauf folgte Wilhelm Busch vor Heine, Erhardt und Jandl. Sicher: Die fünf Szenen waren als lockere Serie von Bildern gedacht, die keinen inneren Zusammenhang aufwiesen. Dennoch schien es uns vorteilhaft, 
Rezitation und Pantomime in einen Rahmen einzufügen. Da es ohnehin geboten war, dass ich als verantwortlicher Dozent ein paar einführende Worte an das Publikum richtete, kamen wir auf die zündende Idee, diesen Vorspann kurzerhand ins Spiel zu integrieren. Ich betrat also die Bühne in der Rolle des Theater-Impresario, der sich mit seiner Schauspieltruppe auf Welttournee befindet. Mich irrtümlich in der Metropolitan Opera in New York wähnend, begrüßte ich die $\mathrm{Zu}-$ schauer in englischer Sprache. Meine verzweifelte Regieassistentin, natürlich eine meiner brasilianischen Studentinnen, hatte alle Hände voll zu tun, um ihrem konfusen Chef klarzumachen, dass er sich weder in der Met noch im Teatro Colón von Buenos Aires, sondern eben, nun ja - im Auditorium der Faculdade de Letras der Universität von Porto Alegre befinde.

Diese beiden Gestalten, der zerstreute Regisseur und seine emsig bemühte Assistentin, kehrten im Verlauf des Stückes noch zwei Mal wieder. Nach der BuschPantomime versuchen sie den Darsteller des Wildschweins, durch die Rolle des Gewaltopfers schwer traumatisiert, von der Bühne zu holen. Die Assistentin redet mit Engelszungen auf ihn ein, er aber bleibt regungslos liegen. Zu ihrem Entsetzen nimmt der Chef die Sache selbst in die Hand: Erst behutsam, dann immer lauter, zuletzt brüllend wie ein Zerberus jagt er den Akteur von der Bühne. Dies ist, falls dieser Begriff existiert, eine interkulturelle Pointe. In dem Stereotyp, dass den Deutschen unverblümte Direktheit kennzeichne, während der Brasilianer seine höfliche Fassade stets beibehalte, steckt ein Funken Wahrheit. Dagegen ist die unter Brasilianern verbreitete Annahme, der Deutsche schreie aus voller Kehle, sobald ihm irgendetwas missfalle, natürlich bloßes Vorurteil. Als solches sollte sie hier auf die Schippe genommen werden.
Ganz zuletzt kommen der Regisseur und seine Assistentin noch einmal auf die Bühne zurück, um sich vom Publikum zu verabschieden. Der Regisseur bittet die Zuschauer um Applaus für seine Schauspieler, falls ihnen das Stück ein wenig gefallen habe. Ich brauche nicht zu erwähnen, dass das Auditório Celso Luft meiner Aufforderung großzügig nachkam.

\section{Resümee}

Die Jornada dos Estudos Germanísticos der Deutsch-Abteilung der UFRGS hatte gewiss interessante linguistische, literaturwissenschaftliche und sprachdidaktische Vorträge zu bieten. In bleibender und zum Glück positiver Erinnerung, dies darf ich in aller Bescheidenheit feststellen, ist den Studierenden und Kollegen jedoch vor allem unsere Theateraufführung geblieben. Das schmeichelt sicherlich dem Ego, wichtiger ist aber, dass es die Außenwirkung der Abteilung innerhalb des Gefüges der sprach- und literaturwissenschaftlichen Fakultät verstärkt. Darüber hinaus scheint mir, dass meine Rechnung auch in didaktischer Hinsicht aufgegangen ist: Nicht nur die Mitwirkenden des Projektes Fünf lyrische Pantomimen scheinen begriffen zu haben, dass sich die Auseinandersetzung mit Gedichten zum Zweck des Spracherwerbs, aber auch um ihrer selbst willen lohnt. Gerade während der Sprechproben und der Arbeit an den Übersetzungen für das Programmheft kam es zu aufschlussreichen Gesprächen, die zwanglos von semantischen $\mathrm{zu}$ phonologischen, von metrischen $\mathrm{zu}$ literaturgeschichtlichen Aspekten führten, ohne jemals den angestrengten Charakter einer Unterrichtseinheit anzunehmen. Zweifellos stimmt es, dass das Verständnis von fremdsprachigen Gedichten ein höheres Maß an Motivation und Interesse erfordert als dasjenige von Lehrbuchtexten. Sie sind 
nun einmal nicht auf den jeweiligen Kenntnisstand des Lernenden abgestimmt und entziehen sich durch ihre konzentrierte Sprache dem leichten $\mathrm{Zu}$ griff. Doch offenbart sich in der Lyrik die eigentliche Essenz einer Sprache. Im DaF-Unterricht darf sie daher auf keinen Fall fehlen.

\section{Literatur}

Brecht, Bertolt: Gesammelte Werke in 20 Bänden, Band 9: Gedichte 2. Frankfurt a. M.: Suhrkamp, 1967.

Burdorf, Dieter: Einführung in die Gedichtanalyse. Stuttgart; Weimar: Metzler, 1997.

Busch, Wilhelm: Historisch-kritische Gesamtausgabe in vier Bänden. Hrsg. von Friedrich Bohne. Wiesbaden; Berlin: Vollmer, 1960.

Dallapiazza, Rosa-Maria; Jan, Eduard von; Schönherr, Til: Tangram. Deutsch als Fremdsprache. Ismaning: Hueber, 1998. 2. Auflage 2002. 4 Bände.

Erhardt, Heinz: Das Große Heinz Erhardt Buch. Hannover: Fackelträger, 1970.

Erhardt, Heinz: Noch'n Gedicht. Oldenburg: Lappan, 2008 (Nachdruck von 1963).

Esselborn, Karl: »Interkulturelle Literaturdidaktik«. In: Wierlacher, Alois; Bogner, Andrea: Handbuch interkulturelle Germanistik. Stuttgart; Weimar: Metzler, 2003, 480-486.

Gernhardt, Robert: Gesammelte Gedichte 1954-2004. Frankfurt a. M.: Fischer, 2005, 3. Auflage Juli 2006.

Gernhardt, Robert: Zur Heiterkeit bereit? (12.02.2004) http://www.faz.net/s/ Rub1DA1FB848C1E44858CB87A0FE6A D1B68/Doc E3F9DD94E0D0D4C87A4C5 343A9FD7FE4D ATpl Ecommon Scontent.html [24.05.2008].

Grünefeld, H. C.: Revolution und Revolutionslieder in Mannheim 1848-1849. Mannheim: Welz, 2006.

Hadulla, Werner: Was ist ein komisches Gedicht? (2005) http://www.klapphornclique.de/seiten/was-ist.htm [24.05.2008].

Heine, Heinrich: Historisch-kritische Gesamtausgabe der Werke. Hrsg. von Manfred Windfuhr. Hamburg: Hoffmann \& Campe, 1973-1997, 16 Bände.
Hoffmann, Heinrich: Der Struwwelpeter oder lustige Geschichten und drollige Bilder für Kinder von 3-6 Jahren. Frankfurt a. M.: Rütten \& Loening, 1858 [1845, 400. Auflage 1917].

Huber, Ruth: Im Haus der Sprache wohnen. Wahrnehmung und Theater im Fremdsprachenunterricht. Tübingen: Niemeyer, 2003.

Jandl, Ernst: Laut und Luise. Hrsg. von Helmut Heißenbüttel und Otto F. Walter. Olten/Freiburg i. Br.: Walter, 1966.

Jacobs, Steffen (Hrsg.): Die komischen Deutschen. Gewitzte Gedichte aus 400 Jahren. 3. Auflage. Frankfurt a. M., 2004.

Link, Heiner: Eine Laus im Uhrgehäuse. Vorwort (2001). http://www.heinerlink.de/ lausvor.htm [24.05.2008].

Mittelstraß, Jürgen: »Die Stunde der Interdisziplinarität? «. In: Kocka, Jürgen (Hrsg.): Interdisziplinarität. Praxis - Herausforderungen - Ideologie. Frankfurt a. M.: Suhrkamp, 1987, 152-158.

Morgenstern, Christian: Gesammelte Werke in einem Band. Hrsg. von Margareta Morgenstern. München: Piper, 1965.

Nünning, Ansgar: »Literatur, Mentalitäten und kulturelles Gedächtnis: Grundriß, Leitbegriffe und Perspektiven einer anglistischen Kulturwissenschaft «. In: Nünning, Ansgar: Literaturwissenschaftliche Theorien, Modelle und Methoden. Eine Einführung. Trier: WTV, 1995, 4. Auflage 2004, 173-198.

Ribeiro de Souza, Celeste H. M.: Retratos do Brasil. Hétero-imagens literárias alemãs. São Paulo: Arte \& Cultura, 1996. http:// www.scribd.com/word/full/529759? access_key=key-rqfv27 fhahfcugn 44ah

Staiger, Emil: Grundbegriffe der Poetik. Zürich: Atlantis, 1946.

Thalmayr, Andreas: Lyrik nervt. Erste Hilfe für gestresste Leser. München; Wien: Hanser, 2004.

Voßkamp, Wilhelm: »Literaturwissenschaft als Kulturwissenschaft «. In: Nünning, Ansgar; Nünning, Vera: Konzepte der Kulturwissenschaften. Theoretische Grundlagen - Ansätze - Perspektiven. Stuttgart; Weimar: Metzler, 2003, 73-85.

Wierlacher, Alois; Bogner, Andrea: Handbuch interkulturelle Germanistik. Stuttgart; Weimar: Metzler, 2003. 
Christoph Schamm

Dr. phil. (Romanistik); Studium der italienischen und portugiesischen Philologie sowie des Faches Neuere Deutsche Literatur an der Ludwig-Maximilians-Universität München. Tätigkeit als Dozent für italienische Literaturgeschichte in München, DAAD-Promotionsstipendiat an der Università degli Studi in Padua,
Italien. Dissertation über Selbstreferenzialität in der modernen italienischen Lyrik. Seit 2006 DAAD-Lektor an der Universidade Federal do Rio Grande do Sul in Porto Alegre, Brasilien. Forschungsund Arbeitsgebiete: Interkulturelle Kommunikation, Lateinamerika-Studien, Lyriktheorien, Literaturdidaktik im DaFUnterricht. 